\title{
Graph Mining Techniques, Tools and Issues - A Study
}

M. Vedanayaki*

\author{
Department of Mathematics, Amet University, Kanathur, Chennai-603 112, India; vedha.m7@gmail.com
}

\begin{abstract}
Data mining has a long history, which has a strong attention from researchers in many different fields including database design, statistics, pattern recognition, machine learning, and data visualization. Data mining is the process of finding projective models from the given data. In this paper we overviewed graph mining tasks and the tools which are used for the mining of data represented as graphs.
\end{abstract}

Keywords: Data Mining, Graphs, Graph Mining, Gephi, Network X

\section{Introduction}

Data mining is the process of the discovery of knowledge from the data. It is a emerging research field of computer science, is defined as the significant process of identifying suitable, prospectively useful, and crucially understandable patterns in data. Graph mining is the process of gathering and analyzing the data represented as graphs ${ }^{4}$. Graph mining has become an important topic of research recently because of numerous applications to a wide variety of data mining problems in biology, chemistry, management, business and communication networking ${ }^{3}$. Graph Mining is a relatively new area of research which however has a solid base in classic graph theory, computational cost considerations, and sociological concepts such how individuals interrelate, group together and follow one another. The structure of the paper is as follows. In Section 2 we discuss the various graph mining techniques and tools. Section 3 confers the issues of graph mining and Section 4 gives the conclusion.

\section{Graph Mining Techniques and Tools}

\subsection{Techniques}

Being a special case of data mining, many data mining approaches have been extended to graph mining also.
The main approaches which are followed for mining of graph data are

- Mining frequent sub graphs

- Classification

- Clustering

\subsubsection{Mining Frequent Sub Graphs}

Frequent sub graphs, as the name suggests, are sub graphs that occur frequently in data represented as graphs ${ }^{1}$. They are useful for characterizing graph sets, discriminating different groups of graphs, classifying and clustering graph sets, building graph indices and facilitating similarity search in graph data bases ${ }^{2}$. A substructure may be different structural forms such as graphs, trees, or lattices, which may be combined with item sets or subsequences. If a substructure occurs frequently, it is called a (frequent) structured pattern.

Although graph mining may include mining frequent sub graph patterns, graph classification, clustering, and other analysis tasks.

\subsubsection{Classification}

Classification is the method of discovering a representation that demonstrates and distinguishes data classes or ideas, for the inspiration to use the model to predict the class of objects whose class label is unknown. The model

*Author for correspondence 
is derived is based on the analysis of a set of training data. There are various other methods for constructing classification models, such as Naive Bayesian classification, support vector machines and $k$-nearest neighbor classification.

\subsubsection{Clustering}

Clustering is under vigorous development. Contributing areas of research include data mining, statistics, machine learning, spatial database technology, biology, and marketing. Owing to the huge amounts of data collected in databases, cluster analysis has recently become a highly active topic in data mining research. Clustering of graphs includes (possibly large) number of graphs which need to be clustered based on their underlying structural behavior. This problem is challenging because of the need to match the structures of the underlying graphs, and use these structures for clustering purposes.

\subsection{Tools}

There are several tools available for graph mining. Some of them are given here.

\subsubsection{Cytoscape}

Is a graph mining tool and it was developed in 2002, with funding from the National Institute of General Medical Sciences and the National Resource for Network Biology. The biomedical research community started using this first, and it is useful to understand the gene and protein interaction in biology.

\subsubsection{Gephi}

Is a open source software and it is suitable for the analysis of all kind of complex networks, but it is mostly used for social network analysis. Classic metrics of social network analysis, such as node degree or between ness centrality measures, can be computed and used in the visualization as well. The network can also be altered based on attributes.

\subsubsection{GraphInsight}

Was once a commercial product, but is now open source. GraphInsight is visualization software that lets you explore graph data through high quality interactive demonstrations. Data exploration and knowledge extraction from graphs is of great interest nowadays.
Knowledge is distributed in social networks, and services are powered by cloud computing platforms. Humans are extremely good in identifying patterns and outliers. GraphInsight is useful for interacting visually with the data can give us a better intuition and higher confidence on the field.

\subsubsection{NetworkX}

Is a Python language package for exploration and analysis of networks and network algorithms Data structures for representing many types of networks, or graphs, (simple graphs, directed graphs, and graphs with parallel edges and self loops). Flexibility ideal for representing networks found in many different fields.

\subsubsection{Social Networks Visualizer}

Social Networks Visualizer also known simply as SocNetV, is a software which is able to compute almost every network property might be interested in, including path lengths, clustering coefficients and graph diameters. Moreover we can also make use of the multitude of layout algorithms provided as well as the random networks that can be quickly generated at the press of a button.

\subsubsection{Knime}

Is a modular platform for building and executing workflows using predefined components, called nodes. Core functionality available for tasks such as standard data mining, analysis and manipulation and extra features and functionality available in KNIME through extensions from various groups and vendors written in Java based on the Eclipse SDK platform.

\section{Issues}

- Scalability

- Data Quality

- Data Ownership and Distribution

- Dimensionality

- Privacy Preservation

- Streaming Data

- Complex and Heterogeneous Data

\section{Conclusion}

In this paper we briefly reviewed the graph mining techniques, tools and issues from its initiation to the 
upcoming research. This paper provides a new perspective of a researcher to overcome the challenges in methods, data and other issues of graph mining in social good.

\section{Acknowledgement}

The author of this article would like to thank AMET University as a team for their full support.

\section{References}

1. Nettleton DF. Data mining of social networks represented as graphs. Elsevier. 2013; 7:1-34.
2. Du H. Data Mining Techniques and Applications an Introduction, 1st Edition. Cengage Learning Edition; 2010.

3. Han J, Kamber M. Data Mining: Concept and Techniques, 2nd Edition. Morgan Kauffmann; 2006.

4. Chen MS, Han J, Yu PS. Data mining: an overview from database perspective. IEEE Transactions on Knowledge and Data Engineering. 1999 Dec; 8(6):866-83. 